\title{
EXPLORING UNDERLYING PATTERNS OF EMERGENT PROBLEM-SOLVING IN CONSTRUCTION WITH TRIZ
}

\author{
Pei-Lun Chang \\ CECI Engineering Consultants, Inc., Taiwan, Taipei, Taiwan \\ Chung Hua University, Hsinchu, Taiwan \\ peilun@ceci.com.tw \\ Wen-Der $\mathrm{Yu}$ \\ Chung Hua University, Hsinchu, Taiwan \\ wenderyu@chu.edu.tw \\ Shao-Tsai Cheng \\ Chung Hua University, Hsinchu, Taiwan \\ shaotsai@chu.edu.tw \\ Shun-Min Lee \\ CECI Engineering Consultants, Inc., Taiwan, Taipei, Taiwan \\ smlee@ceci.com.tw
}

\begin{abstract}
Construction engineers and managers are dealing with emergent problems in their daily works. The problem-solving experiences, usually recorded as historical Lessons-Learned Files (LLFs), accumulated from previous projects are applied repeatedly in similar problems encountered. Such methodology is very similar to the Theory of Inventive Problem Solving (TRIZ) widely adopted in technology innovation fields. The similarity inspires the authors of this paper to develop a preliminary Model of Engineering Problem Solver (MEPS) based on the similar methodology of TRIZ adopted by Altshuller to explore the underlying patterns of problem solving for emergent construction problems. 908 historical LLFs were collected through the Knowledge Management System (KMS) of a top ranked engineering consulting firm in Taiwan. The collected LLFs record the problem descriptions, problem classification, solution description, and the evaluation of the implemented solution, which store the underlying rules or patterns of problem solving that they are ready-for-use by the engineers/managers. By applying the same procedure of TRIZ, a preliminary MEPS is developed with 15 identified management parameters, a contradiction matrix, and 16 problem-solving principles. Two real world problem-solving cases are selected to test the preliminary MEPS. The testing results show great potentials of the proposed MEPS in automated engineering problem-solving. Moreover, the proposed MEPS provides a systematic method for efficient knowledge acquisition and accumulation in engineering consultants.
\end{abstract}

KEYWORDS: Problem-solving, Knowledge management, TRIZ. 


\section{INTRODUCTION}

Construction engineers are faced with various engineering problems in their daily works [1]. In order to resolve the encountered problems, different approaches are adopted including information searching (via libraries, data repository, or internet), expert interviews, and team meetings. Thanks to the advancement of the information technology, the computer based Knowledge Management System (KMS) has been more and more widely adopted by the construction organizations for problem solving [2]. Such a trend is especially true for the engineering consultants in the past decade since the engineering consultants are a knowledgebased industry that generates values trough the knowledge and experience accumulated in the past.

The KMS is a computer program that integrates functionalities including database, community of practice (CoP), and information searching and retrievals. In a traditional KMS, the functions of problem-solving are carried via the so-called CoP, which is a virtual forum formed by a group of members who are interested in the same subject. In a CoP, the members pose subject articles and questions in the virtual forum [3]. The members then respond to the posed subjects and questions by sharing their own perspectives on the subjects or providing their solutions to the questions. The participants of the CoP learn from each other through the recursive processes of knowledge sharing and problem solving. They eventually become the so-called "experts" in the CoP. The experts then apprentice the new members voluntarily to improve the domain knowledge and practical experience of the new members. Since all activities occurs in a CoP are voluntarily, no legal obligations are imposed to the members and the experts who provides knowledge and solutions.

The CoPs were widely employed for problem-solving by engineering consultants in knowledge generation, storing, accumulation, sharing, and management in the past decade due to the adoption of KMSs. Unlike the general CoPs, the members of a CoP in an engineering consulting firm are not completely free of obligations. As a result, the knowledge and solutions provided in the $\mathrm{CoP}$ of an engineering consulting firm are usually carefully prepared and strictly verified by the domain experts before they are shared and disclosed. Moreover, the CoPs provide members with different contexts to intersect and cause the occurrence of so-called "Medici Effects" that speed up the knowledge creation process. Nevertheless, the shared knowledge and solutions are usually the outcomes of knowledge creations by experts based on their valuable practical experiences and expertises that are invaluable to the firm. Some the firms transform the shared knowledge and solutions into a structured database called Lessons-Learned Files (LLFs) [4]. The LLFs are stored in the KMS for reuse by the staffs of the firm. From previous researches, the reuse of LLFs can significantly improve the efficiency of problem solving [5].

The problem-solving approach of LLFs is very similar to the other approach commonly adopted in the field of technology innovation called Theory of Inventive Problem Solving (TRIZ) [6]. The TRIZ method was originally proposed by a former Russian Scientist Genrich Altshuller who investigated more than 200,000 approved patents to develop the model of TRIZ. Altshuller discovered that many patterns are repeated in resolving the problems encountered in inventions. By analyzing the 40,000 most representative patents, he identified 39 representative engineering parameters (EPs) for descriptions of the inventive problems; based on the 39 EPs, he generated a contradiction matrix to explain the conflicts of worsening a set of EPs while an inventor tries to improve a specific EP; finally, 40 inventive principles 
(IPs) are proposed to resolve the previously described conflicts. The TRIZ method has been widely applied to many fields and experienced great successes, including business, social science, architecture, food science, software engineering, micro electronics, quality management, public health, chemistry, biological engineering, operation and service management, education, financial management, marketing, construction engineering, chemical engineering, customer relation management, etc. [7]

The successes of TRIZ inspire the present research to develop a similar problem-solving model for construction engineering since the historical LLFs are repeatedly applied in solving engineering problems. If there exist common patterns of the problem-solving activities in the historical LLFs, the research problem becomes "how can those patterned be identified and in what forms?" In order to resolve the problem, a top ranked engineering consulting firm (CECI Engineering Consultants, Inc., Taiwan) of Taiwan is selected for case study. A complete survey of the 908 historical emergent problem-solving cases recorded in the KMS is conducted. Totally 908 historical LLFs are collected. A research methodology based on TRIZ is adopted to identify the management parameters, contradiction matrix, and problemsolving principles. The reset of the paper is presented in the following: the relevant previous works are reviewed secondly with focus on the lesson-learning methods and the TRIZ method; the methodology of the present research is described thirdly with detailed descriptions of the proposed MEPS and model testing; finally conclusions and future works are addressed.

\section{REVIEW OF RELATED WORKS}

\section{Problem-Solving in Construction}

Problem solving plays the central role of daily construction operations. Li and Love [1] developed a framework of problem-solving for construction engineering and management. Their research identified several characteristics of construction problems that should be tackled in order to solve them quickly, correctly, and cost-effectively, such as the ill-structure nature, inadequate vocabulary, little generalization and conceptualization, temporary multiorganization, uniqueness of problems, and hardness in reaching the optimal solution. Two areas of problem-solving researches tackle the abovementioned issues: the cognitive science and decision support system (DSS). In addition to these two areas, Yu et al. [2] proposed a third approach called Knowledge Management integrated Problem-Solver (KMiPS) to solve emergent construction problems. The KMiPS adopts a KMS and a special designed CoP, namely SOS, for emergent problem solving. Yu et al. proved that the KMiPS achieved both quantitative and qualitative benefits better than the traditional problem-solving approaches. Their research showed that KMS provides desirable functions to tackle the special characteristics of construction problems identified by Li and Love. However, some essential drawbacks exist in the traditional KMS, which may cause poor performance of timeliness and cost effectiveness.

\section{Lessons-Learned System}

An important issue related to construction problem-solving is the compilation of previous learned knowledge that is useful to solve future problems. Such knowledge is usually called "lessons-learned". There have been many existing lessons-learned systems reported in literature, which provides references for the present research. The Hypermedia 
Constructability System (HCS) was developed in collaboration between the Indiana Department of Transportation (INDOT) and the Purdue University [8]. The HCS stores historic lessons-learned in multi-media format so that construction engineers can learn from previous lessons more effectively. The Constructability Lessons Learned Database (CLLD) \& Integrated Knowledge-Intensive System (IKIS) were developed by Kartam and Flood [9][10] to provide a repository for previously learned lessons. The major difference between CLLD \& IKIS and the abovementioned lessons-learned systems is that the latter verifies historic lessons-learned by the domain experts before storing in the database.

The Construction Industry Institute (CII) developed a Lessons-Learned Wizard (LLW) with the package of constructability program [12]. The LLW is a computer aided information system that helps the engineers to record and retrieve the lessons learned from historic projects. The major components of a lesson-learned file (LLF) captured by LLW consists of: (1) problem description-describing the problem encountered in construction process; (2) information of the LLF writer and approvers-providing contacting information for further consultation; (3) solution description-describing the technical and procedural details of problem resolution; (4) evaluation of solution-assessment of the effects and benefits resulted from the lesson-learned. Compared with the other methods mentioned above, the CII's LLF is more suitable for construction problem-solving due to two reasons: (1) more technical and procedural details are provided so that it is easier for users to apply the LLF; (2) the LLFs are verified and assessed before compilation, so that the solution stored in the LLF is more reliable and practical.

\section{The Theory of Inventive Problem Solving (TRIZ) for Construction}

TRIZ was developed by Genrich Altshuller and his colleagues in the former USSR starting in 1946, and is now being developed and practiced throughout the world [7]. Altshuller believed that traditional process for increasing creativity suffers a major flaw that their usefulness decreases as the complexity of the problem increases. Altshuller determined to improve the inventive process, which led to the creation of TRIZ [13]. He studied more than 400,000 patents while deriving this theory. The TRIZ method has been widely applied to many fields and experienced great successes, including business, social science, architecture, food science, software engineering, micro electronics, quality management, public health, chemistry, biological engineering, operation and service management, education, financial management, marketing, construction engineering, chemical engineering, customer relation management, etc. [7]

A very relevant research was conducted by Mohamed and AbouRizk to develop a knowledge representation schema for construction problem solutions (lessons-learned) [11] based on TRIZ [6]. Their schema consists of three major components: (1) the main functions/effects of the solution; (2) the contradiction set of the encountered problem; (3) the resolution principle that best represents the solution. Mohamed and AbouRizk also developed a computer system to implement the proposed schema. Their method provides a framework for efficient knowledge representation for construction lessons-learned. One weakness of the schema is that only principles but no details of problem resolution lessons are stored, which may cause difficulty of users to reapply the lessons-learned. A recent work conducted by Zhang et al., which applied TRIZ to generate innovative ideas in the idea-generation stage of the five-step job plan for value engineering (VE) [14]. Zhang et al.'s research explores the capability of TRIZ in systematic generation of VE alternatives given the objective of improving function 
and reducing cost for the defined problem. Such an application is highly related to the present research that aims at solving a defined problem with previously developed model, except that the present research emphasizes on the special characteristics of construction problems and develops its own models rather than adopting the models of the original TRIZ.

\section{RESEARCH METHODOLOGY}

In light of the similarity between LLFs and TRIZ, the methodology of TRIZ is adopted for the present research. In order to acquire historic LLFs, a local top ranked engineering consulting firm, CECI Engineering Consultants, Inc., Taiwan is selected for case study. Then a six-step research procedure is conducted including: (1) Classification of construction engineering problems; (2) Analysis and selection of representative historical LLFs; (3) Identification of management parameters; (4) Identification of typical problem-solving principles; (5) Establishment of contradiction matrix; (6) Proposing the problem-solving procedure of Model of Engineering Problem Solving (MEPS).

\section{Selected Case Engineering Consulting Firm}

The CECI is one of leading engineering consulting firms in Taiwan. It was established in 1969 primarily for the purpose of promoting Taiwan's technology and assisting in the economic development of Taiwan and other developing countries. The number of full-time staffs of the firm is about 1,700. Among those around 800 are in-house staffs in headquarter located in Taipei, the other 900 are allocated in branches and site offices around the island. Headquarter, braches, and site offices are connected by Intranet.

The structure of the case A/E firm consists of five business groups: (1) Civil Engineering Group; (2) Railway Engineering Group; (3) Electrical and Mechanical Engineering Group; (4) Construction Management Group; and (5) Business and Administration Group. Each business group includes several functional departments. The annual revenue of case $\mathrm{A} / \mathrm{E}$ firm is around 4 billion TWD (128 million USD). According to the information disclosed by the firm, more than 1,700 A/E projects were finished in the past thirty years. Totally volume (construction budget) of the finished projects exceeds 300 billion USD.

\section{Model Development}

\section{Step (1): Classification of construction engineering problems}

The first step of the model development is to classify the 908 historical LLFs. The classification is conducted in two stages: Stage one--classifying the LLFs into engineering problems (805 LLFs) and non-engineering problems (103 LLFs); Stage two--classifying the LLFs different technical categories. The 805 engineering problems are classified into 7 technical categories including: (1) Specification (256 LLFs); (2) Construction technology and management (244 LLFs); (3) Material testing (102 LLFs); (4) Contract (78 LLFs); (5) Vendor information (61 LLFs); (6) Definition of terms (37 LLFs); (7) Information technology (27 LLFs).

After careful reviews of the 805 cases, 322 LLFs (including 244 construction technology and management LLFs and 78 contract LLFs) are found to be the most typical and representative. They are selected for further analysis (see Table 1). 
Table 1: Classification of the 908 LLFs

\begin{tabular}{cccc}
\hline Problem Type & Technical category & Frequency & Selected for further analysis \\
\hline Engineering & Specification & 256 & \\
\hline Engineering & Construction technology and management & 244 & $\mathrm{~V}$ \\
\hline Engineering & Material testing & 102 & $\mathrm{~V}$ \\
\hline Engineering & Contract & 78 & \\
\hline Engineering & Vendor information & 61 & \\
\hline Engineering & Definition of terms & 37 & \\
\hline Engineering & Information technology & 27 & \\
\hline Non-engg. & irrelevant & 4 & \\
\hline Non-engg. & incomplete & 99 & \\
\hline & Summation & 908 & \\
\hline
\end{tabular}

\section{Step (2): Analysis and selection of representative historical LLFs}

The selected 322 LLFs are further analyzed. Initially, the 39 EPs of TRIZ were adopted to characterize the engineering problems. It was realized soon that the EPs of TRIZ are not appropriate for characterizing the construction engineering problems. As a result, it was determined to identify the specific parameters for the engineering problems.

\section{Step (3): Identification of management parameters}

After careful analysis, 15 management parameters (MPs) were identified to replace the 39 EPs in the original TRIZ including: (1) Tendering document; (2) Bidding; (3) Contract; (4) Insurance and bonding; (5) Estimation; (6) Resource; (7) Specification; (8) Productivity; (9) Safety; (10) Material management; (11) Environment and health; (12) Schedule; (13) Cost; (14) Quality; (15) Dispute. The identified 15 MPs are grouped according to the project lifecycle as shown in Table 2.

Table 2: 15 Management parameters (MPs)

\begin{tabular}{cccccc}
\hline Project phase & ID & MP & Project phase & ID & MP \\
\hline Initialization & 1 & Tendering document & Execution & 9 & Safety \\
\hline Initialization & 2 & Bidding & Execution & 10 & Material management \\
\hline Initialization & 3 & Contract & Execution & 11 & Environment and health \\
\hline Initialization & 4 & Insurance and bonding & Control & 12 & Schedule \\
\hline Planning & 5 & Estimation & Control & 13 & Cost \\
\hline Planning & 6 & Resource & Control & 14 & Quality \\
\hline Planning & 7 & Specification & Closure & 15 & Dispute \\
\hline Execution & 8 & Productivity & & & \\
\hline
\end{tabular}




\section{Step (4): Identification of typical problem-solving principles}

In this step, the typical problem-solving principles (PSPs) are identified by reviewing the 322 LLFs. Totally 16 PSPs are most employed by the domain experts, including: (1) Two-party negotiation; (2) Three-party negotiation; (3) Reviewing and reprocessing; (4) Arbitration; (5) Approved by client; (6) Referring to specification; (7) Referring to regulation; (8) Calculated with formula; (9) Referring to contract; (10) Contract change; (11) Design change; (12) Schedule extension; (14) Expert judgement; (15) Historical data; (16) Published database.

\section{Step (5): Establishment of contradiction matrix}

Mimicking TRIZ's contradiction matrix, a similar matrix is established by carefully analyzing the selected 322 LLFs and the associated solutions provided by the domain experts. For example, the problem description of one LLF states: "How to deal with the situation that the contracted specification conflicts with the most updated version of specification published by the government?" The improved MP is "(7) Specification" and deteriorated MP is "(3) Contract". The solution documented in the LLF stated that "It is recommended to initiate the problem by the contractor. The raised problem is transferred to the designer for clarification and solution. If the problem is unsolved, the final decision was made by the client", thus the PSP is referred to "(5) Approved by client". The PSP is then recorded in the contradiction matrix as shown in Table 3. All 322 historical LLFs were reviewed and the associated PSPs were recorded in the contradiction matrix. In some cases, a LLF may associate with more than one PSP. In such cases, all relevant PSPs are recorded. The resulted contradiction matrix is shown in Table 3.

Table 3: Contradiction matrix of MEPS

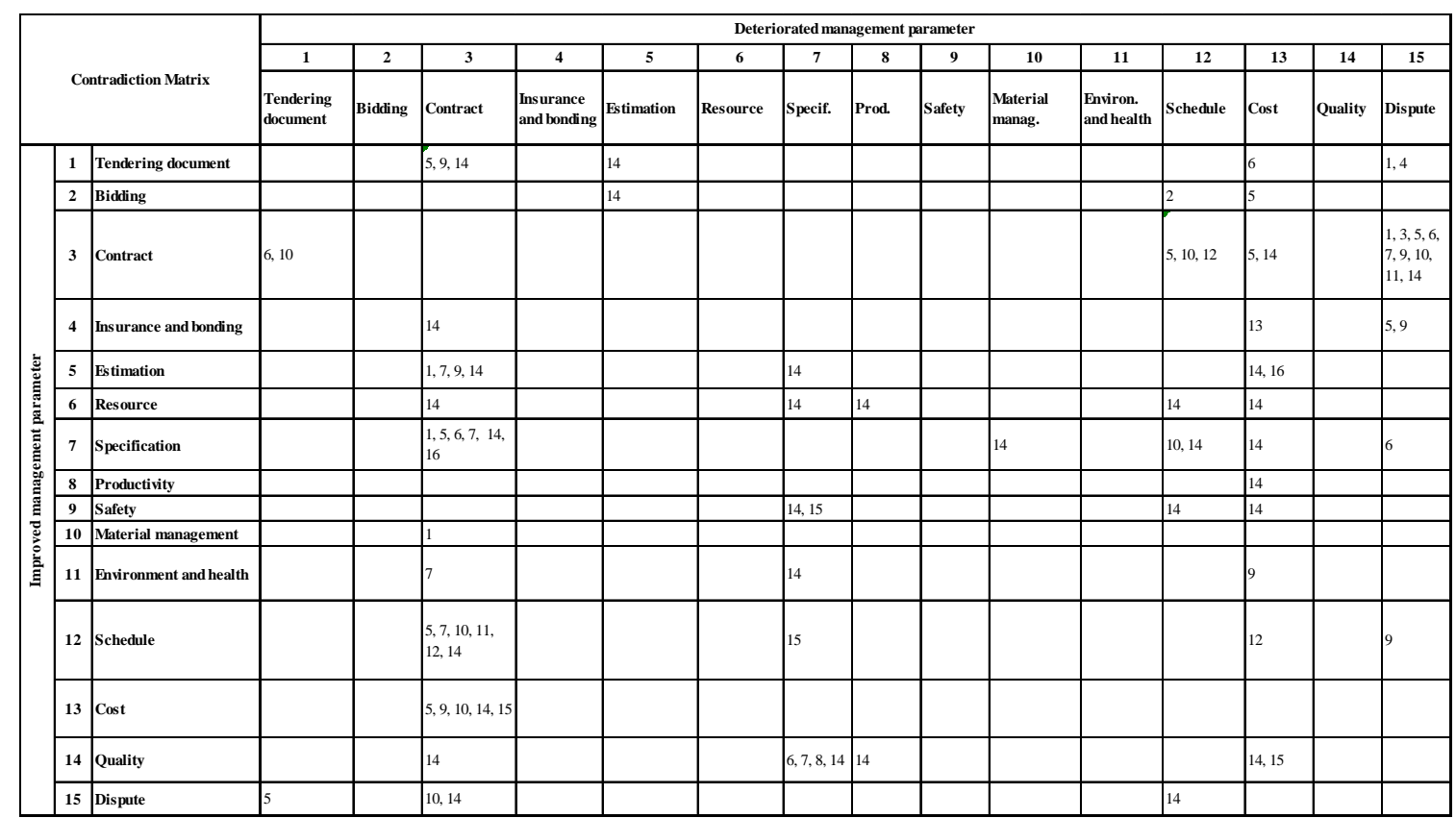

\section{Step (6): Proposing the problem-solving procedure for MEPS}

A problem-solving procedure is proposed for MEPS as shown in Figure 1. The procedure consists of five steps: (1) Analyzing the characteristics of the posed problem; (2) Identifying 
the improved MPs and the deteriorated MPs; (3) Consulting the contradiction matrix to look up the suggested PSPs; (4) Developing final solution; (5) If the solution is resolved, documenting the experience as a new LLF; otherwise, going back to Step (3) and trying out another PSP.

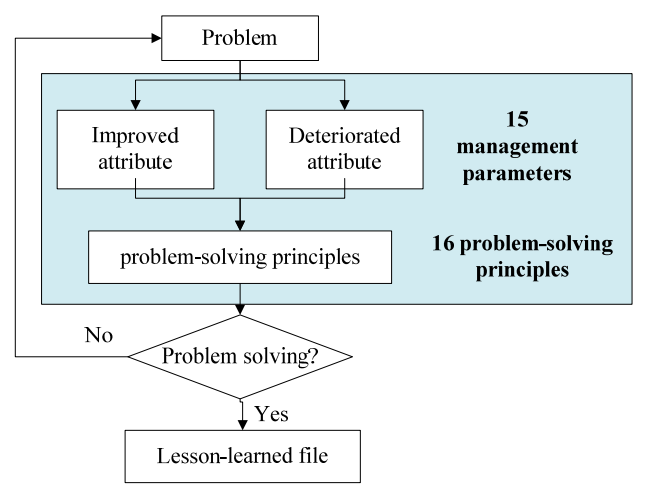

Figure 1: Proposed problem-solving procedure for MEPS

\section{Model Testing}

In order to illustrate the application procedure and test the applicability of the proposed MEPS, two real world engineering problems posed in the SOS (but were not included in the 908 historical LLFs) were selected as testing cases. The two problems were analyzed first to identified the improved and deteriorated MPs. Then, the contradiction matrix is consulted to look up the suggested PSPs. The suggested PSPs are compared with the recommended solutions documented in the LLFs. If the recommended solutions match the PSPs, the proposed MEPS is primitively verified.

\section{Testing case (1)}

Problem description: "Please help define the upper and lower limits of the earth pressuremeter. How to determine the warning limit with respect to variation of temperature? Is it influenced by the geological conditions?"

Improved MP: "(6) Resource"

Deteriorated MP: "(7) Specification"

Problem-solving principle: "(14) Expert judgement"

The documented solution in LLF states: "The variation of the prestressed or axial bearing capacities of the earth should not be influenced by the type of earth. The only exception is for clay. The lateral pressure of clay will be increased as the time of loading passes by. However, the pressure value becomes constant after one week of loading. Such a situation is not applicable for sand-type earth for that the lateral pressure is not affected by loading time. It is noted that the monitoring devices are likely to be damaged during construction operations. As a result, the readings of monitoring devices should be carefully judged by the experienced professional engineers to avoid misunderstanding of the values. According to Taipei MRT project, the values of limits were 125\% (upper limit) and 90\% (lower limit), respectively, of the design value." 
Explanation of testing result: The recommended solution of the LLF is based on the experience of the domain expert. Thus, it matches with the suggested PSP: "(14) Expert judgement." As a result, the recommended PSP of MEPS is validated.

\section{Testing case (2)}

Problem description: "While estimating the connection cost of utility pipeline construction works, how to calculate the extension cost of pipeline of the road side? Should it be included in the cost of the adjunct road section or the extension section? Should it be classified regarding to the existing drainage, building, or the manhole?"

Improved MP: "(5) Estimation"

Deteriorated MP: "(7) Cost"

Problem-solving principle: "(14) Expert judgement" and "(16) Published database"

The documented solution in LLF states: "The calculation of connection cost for utility pipelines should take into account many factors. It should consider the type of refill material, the partition boundary of public and private regions, the budgeting method, the basis of judgement, etc. It is not appropriate to adopt the same standard for all conditions. It is recommended to calculate the costs according to the design drawings and contract specification. The other key point in calculating the cost is that when the extension section is used for motor way and should be paved as it was, it should be included in the adjunct road section. On the contrast, when the extension section is used for pedestrian way and should be paved as it was, it should be included in the extension section."

Explanation of testing result: The recommended solution of LLF refers to the experience of the domain expert and the standard of the current practice, thus it matches with the suggested PSP: "(14) Expert judgement" and (16) Published database". As a result, the recommended PSPs of MEPS are validated.

\section{CONCLUSIONS}

The engineering consulting firm is a knowledge-based industry. The past knowledge and experiences play a very important role in solving future problems. Previous researches adopted knowledge based decision support systems or cognition theory for problem solving. Such methods are not efficient for emergent engineering problems. This paper presents a new approach for engineering problem solving based on a similar method as the Theory of Inventive Problem Solving (TRIZ). The 908 historical lesson-learned files of the selected case engineering consulting firm were collected and analyzed to develop a preliminary Model of Engineering Problem Solving (MEPS). The proposed MEPS consists 15 management parameters, 16 problem-solving principles, and a contradiction matrix. An application procedure is suggested for the proposed MEPS. Two real world engineering problems are selected for illustration of the application and testing of the validity of the proposed MEPS. The testing results show that the proposed MEPS is of great potentials to provide a new approach for engineering problem solving.

The preliminary model of the proposed MEPS has been developed and preliminarily tested. More applications of the proposed MEPS and further verifications of the proposed model will be conducted by the research team in the future. 


\section{ACKNOWLEDGEMENT}

The valuable case study information presented in this paper was provided by CECI, Taipei. The authors would like to express sincere appreciations to the staffs of the Department of Business and Research and Department of Information Systems, CECI Engineering Consultants, Inc., Taiwan, Taipei, Taiwan.

\section{REFERENCES}

[1] Li, H. and Love, P. E. D. (1998). "Developing a theory of construction problem solving," Construction Management and Economics, 16(6), 721-727.

[2] Yu, W. D., Lin, C. T., Yu, C. T., Liu, S. J., Luo, H. C., and Chang, P. L. (2007). "Integrating emergent problem-solving with construction knowledge management system,” Proceedings of the CME 25 Conference, July 16 18, 2007, University of Reading, Reading, UK, 10 pp.

[3] Wenger, E. C. and Snyder, W. M. (2000). "Communities of Practice: The Organizational Frontier,” Harvard Business Review, January-February 2000, pp. 139145.

[4] Fisher, D., Deshpande, S., and Livingston, J. (1998) Modelling the Lessons Learned Process, Research Report 123-11, Construction Industry Institute, Austin, TX, USA.

[5] Yu, W. D., Chang, P.L. and Liu, S.J. (2006). "Quantifying Benefits of Knowledge Management System: A Case Study of an Engineering Consulting Firm,” Proceedings of International Symposium on Automation and Robotics in Construction 2006 (ISARC 2006), Edited by Kano, N. and Arai, T., Session A4—Planning and Management (1), Oct. 3 5, 2006, Tokyo, Japan, pp. 124 129.

[6] Altshuller, G. (1998). “ 40 principles: TRIZ keys to technical innovation.”, L. Shulyak and S. Rodman, trans., Technical Innovation Centre, Worcester, Mass, USA.

[7] The TRIZ Journal, web site: http://www.triz-journal.com, visited 2010/01.

[8] McCullouch, B., and Patty, B., (1994). "Hypermedia constructability system," Proceedings Computing in Civil Engineering (New York), ASCE, New York, NY, USA., n 2, 1397-1404.

[9] Kartam, N., and Flood, I. (1997). "Constructability feedback systems: Issues and illustrative prototype,” Journal of Performance of Constructed Facilities, ASCE, 11(4), 178-183.

[10] Kartam, N. (1994). “Knowledge-intensive database system for making effective use of construction lesson learned,” Proceedings Computing in Civil Engineering (New York), ASCE, New York, NY, USA., n 2, 1139-1145.

[11] Mohamed, Y. and AbouRizk, S. (2005). “Technical knowledge consolidation using theory of inventive problem solving,” 131(9), 993-1001.

[12] Construction Industry Institute (1993). "Constructability implementation guide," Publication 34-1, Austin, TX, U.S.A.

[13] John Terninko, Alla Zusman, Boris Zlotion. "Systematic Innovation- An Introduction to TRIZ.”, CRC Press,1998.

[14] Zhang, X., Mao, X., and AbouRizk, S. M. (2009). Developing a knowledge management system for improved value engineering practices in the construction industry, Automation in Construction, 18, 777-789. 